\title{
Comparison of the Development of TikTok and Bilibili
}

\author{
Yiting Sun* \\ Southwest Weiyu Middle School, Shanghai, China \\ *Corresponding author: Yiting Sun, lynnlin@xyzrgroup.com
}

\begin{abstract}
According to the comparison between TikTok and Bilibili in five aspects, TikTok is far more than Bilibili in terms of user flow and business model. However, TikTok still has some disadvantages, such as the failure to provide high-quality short videos or the lack of development prospects for creators. TikTok and Bilibili are two related but different video platforms. Through the comparison between them, we can intuitively understand the problems faced by the two companies and predict the developmental trend of the two in the future. People can clearly understand the development and future trend of entertainment video platforms through the comparison between the two. The author thinks, TikTok will continue to generate high revenue with high traffic, and if Bilibili continues to produce high-quality videos and tries to break into the market, it will be able to gain a foothold like Youtube.
\end{abstract}

Keywords: TikTok; Bilibili

Publication date: July 2021; Online publication: July 31, 2021

\section{Introduction}

In 2021, the number of people using the Internet worldwide has reached 4.66 billion. In the Internet era, there is an increasing number of people use various online platforms for social entertainment. TikTok, the leading social software for short videos, has seen its active users grow from 250 million in 2019 to 400 million in 2021. However, Bilibili, another popular video website among young people, has also attracted the attention of a large number of people, and the number of active users has surpassed the iQiyi, Tencent, and other well-known video software. In order to study their development differences and future development prospects, the author made an analysis based on the audience, main business content, business model, ecological differences between creators, and the barriers between Bilibili and TikTok. The conclusion is that for enterprises, TikTok will always win with high traffic and high profit, but from the perspective of creators of the two platforms, B has greater potential.

\section{Data and Method}

\subsection{The audience of TikTok and Bilibili}

Bilibili is currently trying to develop out of the wall of the quadratic elements. The users are mainly divided into two main bodies: the core users of the quadratic elements and the general quadratic element users. The users are also divided into three groups according to their functions in Bilibili. First group is contentcurators, the core members of Bilibili, who carry or self-produce videos and upload them to the platform for everyone to watch to get revenue. The second group is internet anchors who get profits through live broadcasting and interaction with viewers. The third group is video consumers, the main source of benefits for content-curators and anchors, who browse and watch ordinary videos and live broadcasts, and providing likes, coins, shares, comments, etc. Bilibili is a platform highly gathered by the young generation. About 
$60 \%$ of the users are $20-29$ years old, and the rest of the 30 -plus age group is no more than $15 \%{ }^{[1]}$. In addition, there is a gender imbalance of Bilibili, women make up 30 percent of the total, while men make up a staggering 70 percent. Besides, Bilibili's main users are in the first and second tier cities in the coastal region and a few central cities. Nearly $70 \%$ of users are from first tier cities. According to the provinces where the users of Bilibili reside, the top three provinces are Guangdong, Jiangsu and Shandong respectively. The users of Guangdong account for $12.4 \%$ of the total users, and the users of Jiangsu account for $10.2 \%{ }^{[10]}$. TikTok users are categorized into three divisions according its users' functions. The first group is called KOL (Key Option Leader), who were recruited by TikTok company in the early stage and trained for creating videos for their strong desires to express themselves, their high enthusiasm for music, creative video production, editing and appealing audience. The second group is called "follower-type users," who appreciate the talent wonderful works of KOL and are eager to be able to shoot the same cool video in TikTok through several methods step by step: finding their idol in their heart on the platform, following them, learning from them, and participating in the challenging topics of TikTok. The third group is called "browsing users," like video consumers in Bilibili, who watch videos created by KOL or follower-like users and share videos with friends. However, the users of TikTok is more comprehensive and balanced. First, it aims at all ages. The margin of error for all the different age groups after age 19 was between 10 and 20 percent ${ }^{[2]}$. Second, the proportion of male and female users of TikTok is fairly equal. According to the data from QuestMobile, men make up 52\% of the total users and women $48 \%$ of the total users. Third, TikTok is welcomed by users who reside in provinces in wide ranges, from first-tier cities to lower-tier cities, including cities below third-tier. The average error of the number of users from first-tier cities to cities below fifth-tier cities is not large, between 10\%-25\%, among which third-tier and fourth-tier cities account for the highest proportion, accounting for $25 \%$ and $22 \%$ of the total, respectively ${ }^{[11]}$. However, the disadvantage of TikTok lies in its strong randomness of users, while most of the users of Bilibili are loyal users, which is reflected in the high distributions of a large number of core quadratic users.

\subsection{TikTok and Bilibili content}

At the early stage, the content of TikTok videos are various and are constantly diversifying, thanks to the creativity of users. TikTok videos are divided into 14 categories: entertainment, knowledge, quadratic elements, games, food, sports, fashions, dance, music, information, technology, living, funny videos and VLOG. Also, the TikTok is not only popular for Chinese people, because it can cross cultures, classes, and even languages. According to the TikTok download data by countries in $2019^{\text {[3] }}$, TikTok is the fourth most downloaded app globally, just behind Facebook. India has seen 190.6 million downloads, and the US has seen 41 million downloads. TikTok's videos are attracting and make users easily addicted in them because the platform can push corresponding videos according to customers' preferences for watching short videos, ensuring users' sense of experience. Thus, people's acceptance of TikTok videos is quite high. On the other side, Bilibili videos can be clearly divided in to 15 areas: animation, music, dance, games, knowledge, technology, living, ghost and animal, fashions, entertainment, information, films and television, food, funny videos and VLOG. Since Bilibili stands as a quadratic element leading platform for the cultural community and video, so users' interests are more central and limited in quadratic elements, which is the reason why Bilibili are largely gathered by young generations. Bilibili's popularity is largely due to the biggest advantage of no video advertising and content of UGC. The problem is the quadratic-element contents and memes which are prevalent in Bilibili might not be understood and recognized by general groups, who are not familiar with the quadratic-element circle. Therefore, the range and variety of Bilibili users is much smaller than that of TikTok. In attempt to address this problem, Bilbili is taking action to develop in the direction of diversification. In addition to quadratic-element animation, the videos of Bilibili are breaking their way to knwoledge, technology, and vlog-type fields according to the speech of Chen Rui, the founder 
of Bilibili ${ }^{[4]}$. Nevertheless, from the aspects of the quality of videos, TikTok is with disadvantages. What TikTok lacks is that it is difficult to show a 15-second video with high quality. In contrast, videos of Bilibili, usually lengths from 15 seconds to even hours, are all uploaded by professional users (content-curators).

\subsection{The business Model of TikTok and Bilibili}

There are four aspects for analyzing business model of TikTok. Firstly, according to the statistics from CNNIC ${ }^{[12]}$, China's netizens aged 10-39 are the main Internet users, accounting for 73 percent of the total, and the 20-39 age group accounts for up to 20 percent. Douyin meets the spiritual needs of young people through the value proposition of the new generation, enabling them to discover their true inner selves and have a better chance to be recognized by the outside world. Therefore, Douyin is favored by the post-80s, post-90s and post-00s, which brings out potential business values. Secondly, KOL, the main groups of TikTok users who spread their talented creations and performance, are highly imitated and followed by the rest groups of users. Though this method can bring out high traffic, the types of videos in TikTok will gradually appear monotonous, boring, and are easy to produce aesthetic fatigue, as the method limits users' originality. Thirdly, the short video format in TikTok greatly reduces the time cost of users. Actually, from a micro point of view, Tiktok does take up a lot of fragmented time, such as waiting for the subway or bus, or going to bed. As the data shown from iiMedia Research ${ }^{[13]}$, of the 700 million people who use short videos, 37.2 percent of users increase their use of TikTok in 2021, and 31 percent of TikTok users stay the same in 2021. Thus, time spent by the public is on the rise. Fourth, by looking at TikTok's profit model, TikTok makes most of its fortunes from advertising and in-app purchases, as shown by financial statements

${ }^{[5]}$ that reported $\$ 88.5$ million in revenue from advertising and in-app purchases in the fourth quarter of 2019, twice as much as in the third quarter of 2019 and six times as much as in the fourth quarter of 2018. On the other hand, there are three aspects to analyze business model of Bilibili. Firstly, the biggest advantage of Bilibili lies in its core competitiveness. Different from iQiyi, Tencent, Youku and other video websites, Bilibili is like a cultural community in essence, which has cultivated a group of highly loyal users. In addition, Bilibili strengthened the establishment of language within the circle by means of the interaction represented by bullet screen. Site Bilibili members enjoy uploaded video updates, on-demand advertising ways to promote individual users of the site monthly contact. Secondly, Bilibili adopts the method of business combination, mainly focusing on the offline activities of the quadratic element and the gradual development of peripheral products. Bilibili uses labels to mark video contents, making it form a different cultural circle. The fourth aspect is the profit model of Bilibili. In fact. The revenue of Bilibili at the beginning came from Baidu and Google. However, with the continuous expansion of user traffic, the website with huge traffic naturally gives rise to many profit points, which can be roughly divided into games, live broadcasting, advertising and others. Advertising is the earliest income of Bilibili site, and the original position is mainly concentrated in the content page player barrage pool, with multiple game advertisements; Website home page advertising, mostly diffuse and other websites around the piracy; Content page player ads, mostly for most game agencies game ads. Nevertheless, the ads now are eliminated except those from barrage pool, so advertising is no longer the main source of making profits. Now, Bilibili make a fortune largely from games. Because of the vertical concentration of users on Bilibili and the high retention and conversion rate, Bilibili, as a cultural area of two-dimensional aggregation, should not be ignored most definitely is the game in ACG. In addition to promoting the game in the content curators' videos, Bilibili will be jointly operated with the game developers after the invention of the game, and then the revenue will be paid according to the agreed proportion. According to the financial statements from Bilibili ${ }^{[6]}$, Bilibili's profit income from developing and distributing games, is 873 million yuan, accounting for more than $64 \%$ of the total, most of which comes from its sole agency of fate/grand orders ${ }^{[5]}$. Now, version 2.0 of this game also led to a certain growth of the total revenue. Revenue from direct broadcast 
and value-added services reaches 292 million, accounting for $21 \%$ of the total. The remaining $15 \%$ of the revenue comes from advertising, e-commerce, and other income. Consequently, in terms of revenue, TikTok is far ahead of Bilibili, but we can clearly see that the two apps have different operating models and aims, both of which benefits and fit the promotion of these two apps.

\subsection{Ecological differences between TikTok and Bilibili creators}

Compared with each other, in fact, creators in the Bilibili are to benefit more. The platform provided by Bilibili has a large number of loyal users, high user engagement, and high fan activity, but for TikTok, a short 15-second video with a large number of relevant notifications makes it difficult for users to connect

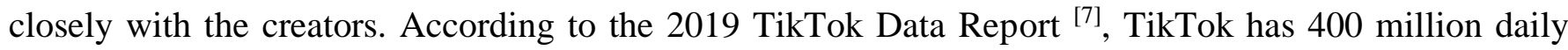
active users. According to the Q3 2019 financial report, TikTok has only 376 million daily active users, which is $9 \%$ of TikTok's total daily active users. However, the point is that $9 \%$ of Bilibili curators contribute $12 \%$ of its followers, indicating that Bilibili has a $33 \%$ higher chance of getting attention from users than TikTok. In addition, the continuous expansion of Bilibili this year ${ }^{[9]}$, from the original main content of quadratic elements to the knowledge area, gives more creators the opportunity and space to show. For example, the knowledge area can be said to be a dark horse in the whole Bilibili this year. Up owner Luoxiang and Crayon and Xiaoxun are good examples, both of whom are followed by more than 5 million fans. In the past two years, however, TikTok has also hatched a strategy for its creator ecosystem. At the TikTok Creators Conference in $2021^{[8]}$, Zhang nan, the CEO of TikTok, said that the platform will hatch a "creator Growth plan" that aims to encourage at least 10 million creators to earn profits from TikTok. In the past two years, the ecosystem of creators has been in a state of growth. Among the creators with tens of thousands of followers on TikTok, $88 \%$ are from ordinary original creators, while among the creators with millions of followers, $85 \%$ are also from ordinary original creators. In addition, TikTok's live-stream delivery to e-commerce has also grown rapidly, as TikTok has lowered the threshold for e-commerce to 1,000 followers. In this year's "515 Ace Live Studio event," more than 1,700 million content creators have participated in the livestream, covering video, beauty, jewelry, clothing, and other fields. As data shows ${ }^{[7]}$, creators on TikTok's chart of celebrities saw their per capita income double. Therefore, to sum up, the loyal users of Bilibili can bring a lot of benefits to creators, while the measures TikTok has implemented in the past two years are also assisting in creators and improving their ecology.

\subsection{The Barriers of TikTok and Bilibili}

TikTok's core value to users is to provide the most efficient means of entertainment, which means that these means of entertainment will not be permanent, because, with the development of The Times, more efficient ways of entertainment that we can't imagine will appear in the future, and which would be the substitute of TikTok. The barrier of Bilibili is the content ecology composed of creators and users. With the increase of new creators and users, the content of videos will also change. For example, many funny memes are actually created by ordinary users. Such baggage and meme will be replaced quickly as time goes by. What Bilibili can do is that it can advocate high-quality videos and accumulates high-quality content like YouTube, Bilibli will be at the same station like it. Most importantly, the expanding of fields of videos of Bilibili will determine whether it can have greater development in the future. Thus, in order to have greater development, TikTok should be more original in providing means of entertainment and Bilibili should expand the variety of its videos.

\section{Results and Discussion}

\subsection{Comparison and Developmental Prospect of TikTok and Bilibili}

If TikTok and Bilibili compete with each other, Bilibili has totally no advantages at all, but the stability and 
liquidity of Bilibili have competitive powers. According to the ecological differences between creators of the two apps, both TikTok and Bilibili have developmental opportunities in different aspects. Bilibili provides creators with a platform with higher fan stickiness and larger creative space, while TikTok provides creators with more training opportunities and spaces for growth. While the main threat to Bilibili is its disruptive process, the threat to TikTok is whether there are new scenarios that can capture users' attention. In terms of the profit model, the profit of TikTok is significantly higher than that of B station. Therefore, Bilibili needs to find new ways to make money, such as vigorously supporting the live broadcasting industry and making a fortune by seizing the advantages of Bilibili, without breaking the cultural value and community atmosphere advocated by Bilibili. Consequently, what has to conclude from the comparison of the two apps is that both of them have their unique features, and TikTok is more dominant, but as seeing both platforms' measures for improvement, their future competition will be unknown and unexpected.

\section{Conclusion}

To conclude, by weighing both TikTok and Bilibili in 5 aspects, TikTok is more dominant than Bilibili, especially indicated by measurements from business model. However, by taking the long view, the author can't evaluate developments of both apps. It is safe to say that both platforms are having attempts to reform and innovate to different ways: Bilibili is expanding its ranges of video varieties and TikTok is assisting content-curators' growth. All in all, we can have good expectation toward both TikTok and Bilibili which might build more glittering things.

\section{Acknowledgments}

What has to be admitted is that it is hard to forecast the future development of TikTok and Bilibili accurately. Statistics for analyzing TikTok and Bilibili before 2018 have not been included in the analyzing part, which may somewhat influence the results.

\section{Disclosure statement}

The author declares no conflict of interest.

\section{References}

[1] Li Y, 2019, Report of Bilibili User Requirements Analysis, October 11th, 2019

[2] QuestMobile, 2020, TikTok User Portrait Report in 2020, Jan.

[3] App Annie, 2019, TikTok Global User Statistics Collection.

[4] Bilibili, 2020, Transcript of Chen Rui's speech, Sept, $28^{\text {th }}$.

[5] Wu Dong, 2019, Unaudited Financial Statements of Bilibili, May, $16^{\text {th }}$.

[6] Jn Niu, 2020, TikTok Data Report from Sensor Tower, Jan, $19^{\text {th }}$.

[7] TikTok, 2019, TikTok Data Report.

[8] TikTok, 2019, Transcript of TikTok Creators Conference.

[9] Ka Si Data, 2020, Bilibili Developmental Analysis.

[10] Jiang Z, 2020, Ranking of Bilibili Users by Provinces, May, $8^{\text {th }}$.

[11] QuestMobile, 2021, Attribute Analysis of Tiktok Users, Jan.

[12] CNNIC, 2020, Statistical Report on Internet Development in China.

[13] iiMedia Research, 2020, Study of Duration of Different Types of Short Video Users. 